FOLIA

Amazónica

Revista del Instituto de Investigaciones

de la Amazonía Peruana

\title{
SITUACIÓN ACTUAL DE LA BASE DE DATOS GENÉTICOS DE VERTEBRADOS DE LA REGIÓN LORETO, PERÚ
}

\author{
Rommel R. ROJAS ${ }^{1}$; Juan C. CASTRO², Carmen GARCIA-DÁVILA ${ }^{3}$ \\ 1 Programa de Post-graduación en Genética, Conservação e Biologia Evolutiva, Instituto Nacional \\ de Pesquisas da Amazônia-INPA, Manaus, Brasil. rrojaszamora@gmail.com \\ 2 Centro de Investigación de Recursos Naturales-CIRNA, Universidad Nacional de la Amazonia \\ Peruana-UNAP, lquitos, Perú \\ 3 Instituto de Investigaciones de la Amazonia Peruana- IIAP, Laboratorio de Biología y Genética \\ Molecular-LBGM, Iquitos, Perú
}

\section{RESUMEN}

La región Loreto presenta la mayor extensión geográfica del Perú y posee una megadiversidad de especies de flora y fauna que da soporte al desarrollo de actividades socioeconómicas y culturales. En este estudio actualizamos el conocimiento de la base de datos genéticos de vertebrados existentes en Loreto. Utilizamos el buscador de secuencias genéticas implementado en el paquete rentrez del programa $\mathrm{R}$, organizamos y comparamos la cantidad de secuencias en diferentes niveles taxonómicos de cada orden de vertebrados, genes más empleados y verificamos la existencia de secuencias genéticas para especies de interés comercial. Los resultados indican la existencia de 1960 secuencias genéticas depositadas en GenBank, de los cuales 38,52\% pertenecen a la Clase Peces; 29,33\% son anfibios; $21,98 \%$ aves; 8,92\% mamíferos y 1,22\% reptiles. Estimamos que solo el 19,44\% de especies de vertebrados en Loreto poseen datos genéticos. Los genes más usados variaron en abundancia dependiendo del grupo de vertebrados. La mayoría de las especies de interés comercial no presentaron datos genéticos. Conocer el panorama de la base de datos genéticos de la región Loreto es indispensable para su integración con estudios ecológicosevolutivos y la elaboración de planes de manejo y desarrollo sustentable.

PALABRAS CLAVE: Amazonía, conservación, ecosistemas, especies, genes. 


\title{
SITUATION OF THE GENETIC DATABASE OF VERTEBRATES OF THE LORETO REGION, PERU
}

\begin{abstract}
The Loreto region has the greatest geographical extension of Peru and has a mega diversity of flora and fauna that supports the development of socioeconomic and cultural activities. In this study we evaluate the knowledge of the genetic database of vertebrates in Loreto. We use the gene sequence search implemented in the $\mathrm{R}$ rentrez package, we organize and compare the quantity of sequences in different taxonomic levels of each order of vertebrates, most used genes and existence of genetic sequences for species of commercial interest and for conservation. Our results indicate the existence of 1960 genetic sequences deposited in the GenBank, of which 38,52\% belong to the Fish; 29,33\% are amphibians; $21,98 \%$ birds; $8,92 \%$ mammals and 1,22\% reptiles. Our results estimates that only $19,44 \%$ of vertebrate species in Loreto have genetic data. The genes most used for these analyzes varied in abundance depending on the group of vertebrates. All species of commercial and conservation interest did not present genetic data. The general panorama of the genetic database of the Loreto region is indispensable for its integration with ecological-evolutionary studies and the elaboration of sustainable management and conservation plans.
\end{abstract}

KEYWORDS: Amazon, conservation, ecosystems, species, genes. 


\section{INTRODUCTION}

La Amazonia se extiende por los países de Guyana, Colombia, Ecuador, Bolivia, Surimane, Guyana Francesa, Brasil y Perú (Mittermeier et al., 1998), representando uno de los últimos refugios de la diversidad biológica del mundo (Fearnside, 2005; Jenkins et al., 2013). Sin embargo, con el incremento sin precedentes de la destrucción de sus frágiles ecosistemas muchas poblaciones de flora y fauna están siendo amenazadas y muchas de ellas aún no han sido descubiertas (Bickford et al., 2007; Fouquet et al., 2007; Gehara et al., 2014).

El departamento de Loreto, Perú posee el 28\% del territorio peruano con una área de 368582 $\mathrm{km}^{2}$ y cuenta con una población aproximada de 883510 habitantes (INEI, 2015). Esta región alberga una enorme riqueza de vertebrados $(\sim 2$ 423), siendo reconocidos 914 especies de aves, 800 especies de peces, 267 especies de mamíferos, 262 especies de anfibios y180 especies de reptiles (Pitman et al., 2010; Ortega et al., 2012). Estos recursos faunísticos son de suma importancia para la alimentación urbana y rural, fuente de renta económica y desarrollo sostenido de la población Loretana. Sin embargo, en los 30 últimos años se encuentran sufriendo una fuerte presión antrópica, producto del crecimiento urbano, aumento de los medios de transporte fluvial, que permite alcanzar lugares antes impensables; y la facilidad en la adquisición de instrumento de caza y pesca más eficientes, que permiten una masificación de estas actividades. Todo esto ha ocasionado un aumento en el grado de contaminación, destrucción de hábitats naturales e impacto sobre los recursos, sumado a los planes de construcción de hidroeléctricas $\mathrm{y}$ al creciente proceso de calentamiento global, no permite augurar la sostenibilidad de estos recursos en esta región (Fearnside, 2006, 1996; García-Dávila et al., 2014, 2015).
Para evaluar los impactos ambientales de los recursos faunísticos es fundamental analizar los patrones de su diversidad genética. Estos análisis representan herramientas poderosas que ayudan a conocer su dinámica poblacional, identificar especies nuevas, inferir procesos biogeográficos y planificar acciones de conservación (Avise et al., 1987; Florio et al., 2012; Cozzuol et al., 2013; Hrbek et al., 2014; Rojas et al., 2016). Estudios de diversidad genética de los recursos faunísticos en la Amazonía peruana y en particular en la región Loreto son escasos, fragmentarios o incompletos, a pesar de ser considerada una región hotspot de la diversidad biológica (Shanee, 2012).

El conocimiento de la diversidad genética en una determinada área geográfica es la base para la inferencia de procesos de adaptación en ambientes cambiantes (Hedrick \& Miller, 1992), consecuentemente, esta investigación se realizó para conocer la situación actual de la información genética disponible de vertebrados de la región Loreto y establecer una línea base para futuros estudios de la diversidad genética a nivel molecular. Para ello se recopilo información genética (secuencias de DNA) de peces, anfibios, reptiles, aves y mamíferos de Loreto depositados en uno de los mas importantes repositorios internacionales de DNA: GenBank (Benson et al., 2013).

\section{MATERIALES Y MÉTODOS}

Utilizamos el buscador de secuencias genéticas del paquete Rentrez (Winter, 2017) del programa R para contabilizar las secuencias de vertebrados (Peces, Amphibia, Reptilia, Aves y Mammalia) de la región Loreto depositados en GenBank (http:// www.ncbi.nlm.nih.gov/Genbank). Los gráficos fueron elaborados usando el paquete ggplot 2 (Wickhan, 2004).

Las búsquedas fueron realizadas en diferentes niveles taxonómicos (Orden, familia, género y 
especie) para cada grupo de vertebrados. Para facilitar la comparación de las secuencias de ADN, sólo se incluyó secuencias obtenidas con el método de Sanger. También, se determinó la abundancia de nucleótidos por tipo de genes mitocondriales (p.ej., subunidad I de la citocromo oxidasa (COI), citocromo B (CytB) y la región Control (D-loop) y genes nucleares (p.ej., gen 1 activador de recombinación (RAG1), tirosinasa, y rodopsina). Adicionalmente, se identificaron secuencias genéticas de especies de interés comercial y alimenticio. Finalmente, se compararon los números de secuencias genéticas de los cuatro departamentos que conforman la Amazonía peruana (Loreto, Ucayali, Madre de Dios y San Martin). Las secuencias consideradas en el análisis fueron las que fueron depositadas y liberadas para uso público hasta el 10 de Setiembre del 2018.

\section{RESULTADOS}

Nuestros resultados muestran la existencia de 1960 secuencias de DNA (Vertebrata + Peru + Loreto) depositadas en GenBank (Figura 1). De estas secuencias $755(38,52 \%)$ son de peces, 575 de anfibios (29,33\%), 431 de aves (21,98\%), 175 de mamíferos $(8,92 \%)$ y 24 de reptiles. $(1,22 \%)$.

La Figura 2 muestra que la Clase Peces presento mayor cantidad de secuencias genéticas en el Orden Cichliformes (666) seguido por Siluriformes (58) y Characiformes (31). La Clase Amphibia presento mayor cantidad de secuencias en el Orden Anura (573), Caudata (2) y ninguna Apoda. Mientras que en la Clase Reptilia solo existen secuencias en el Orden Squamata (24), mientras que los Ordenes Chelonia y Crocodrilia no presentaron secuencias depositadas. La Clase Aves mostro mayor número de secuencias genéticas en la Orden Passeriformes (352), seguido por Piciformes (87), Trogoniformes (7)

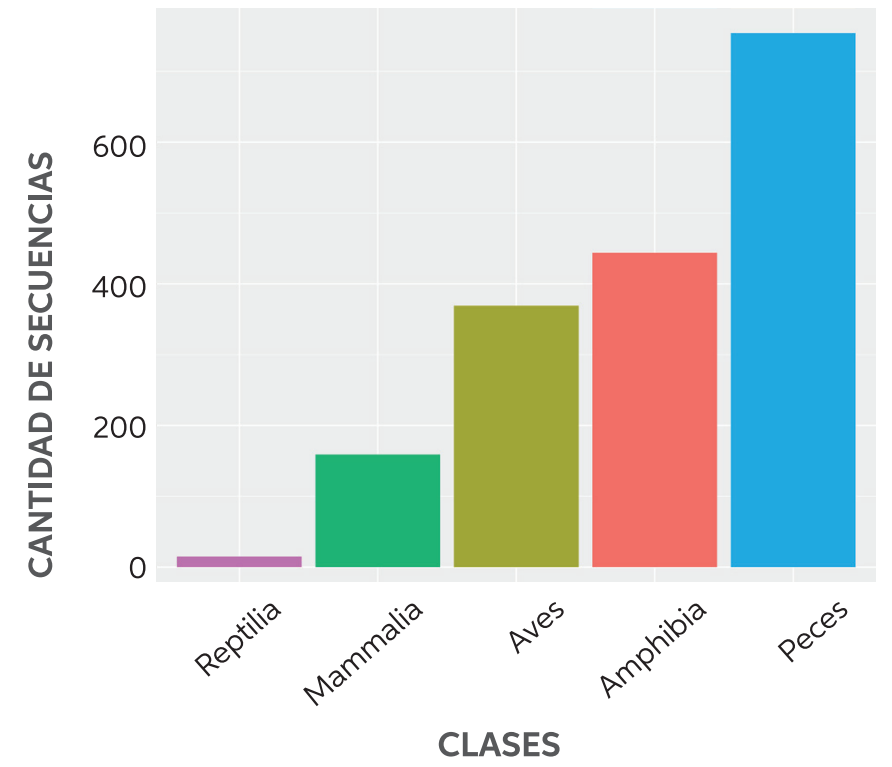

Figura 1: Abundancia de secuencias genéticas de vertebrados en la región Loreto, Perú.

y Strigiformes (6). Dentro de la Clase Mammalia la Orden Didelphimorphia presento mayor número de secuencia genéticas (76), seguido por Rodentia (64), Chiroptera (18) y Primata (12).

Dentro da Clase Peces la familia más representativa fue Cichlidae con 666 secuencias genéticas, seguido por Serrasalmidae (21), Loriicaridae (17) y Characidae (8). La familia con mayor cantidad de secuencias genéticas de la Clase Amphibia fue Dendrobatidae (215), seguido por Bufonidae (164), Hylidae (119), Microhylidae (40), Aromobatidae (29) y Leptodactylidae (16). La Clase Reptilia tuvo mayor representatividad por la familia Gymnophthalmidae (17), seguido por Iguanidae (6) y Elapidae (1). La familia de la Aves con mayor cantidad de secuencias genéticas fue Ramphastidae (80), seguido por la familia Thraupidae (70), Certhiidae (43), Cotingidae (32), Trogonidae (9) y Pipridae (5). La Clase Mammalia tuvo más representatividad por la familia Didelphidae (76), seguido por Cricetidae (64), Noctilionidae (15), Cebidae (8) y Cyclopedidae (5) (Figura 3). 

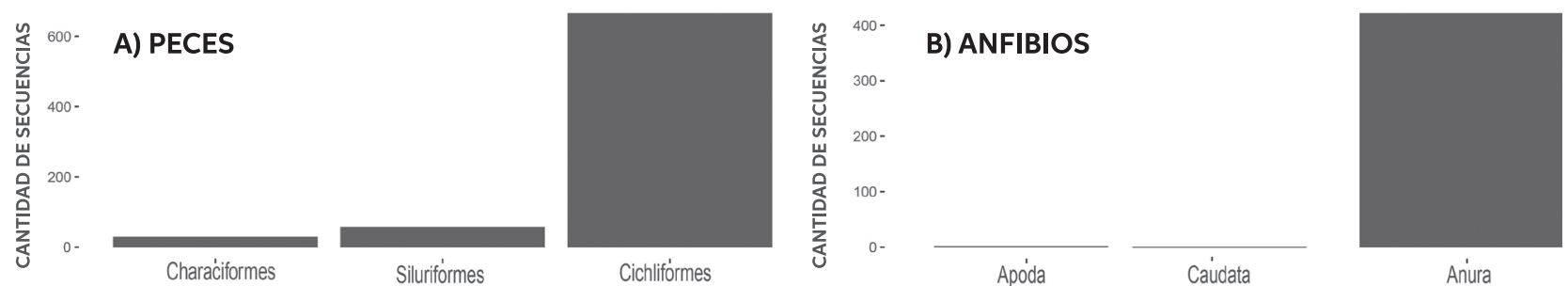

Anura
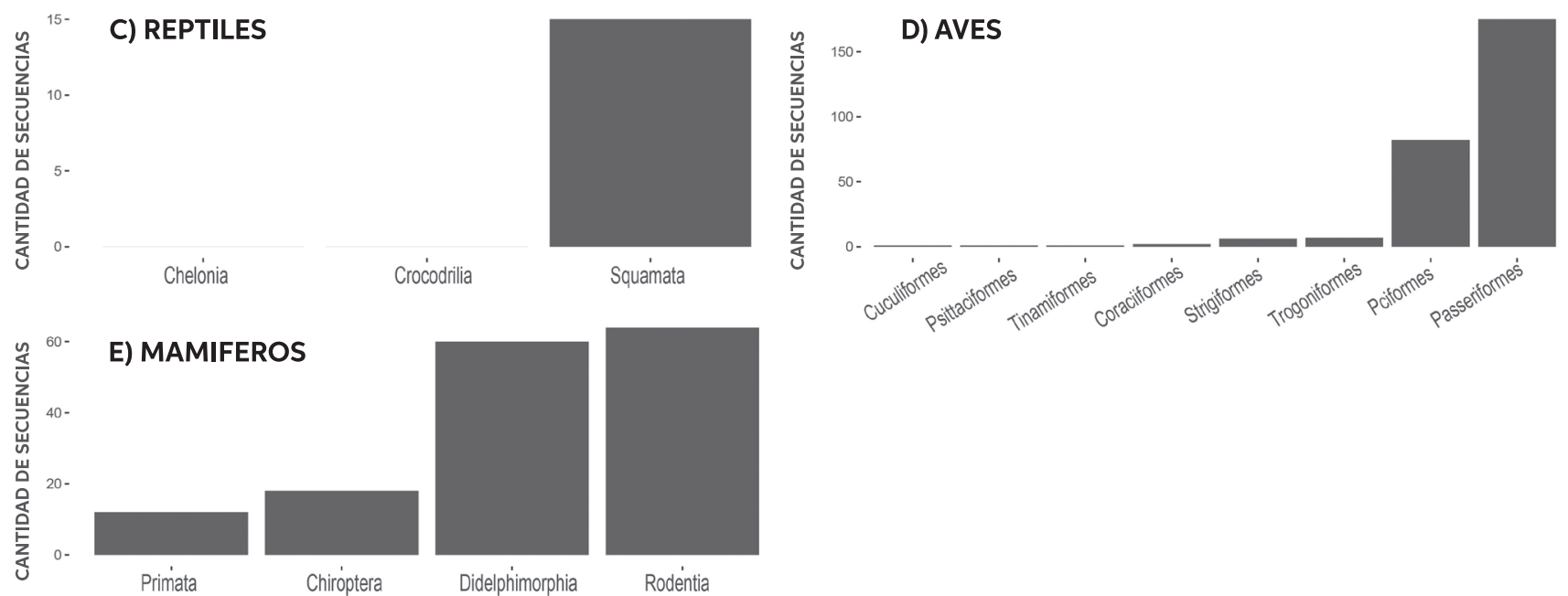

Figura 2: Abundancia de secuencias genéticas por Clases de vertebrados en la región Loreto, Perú.

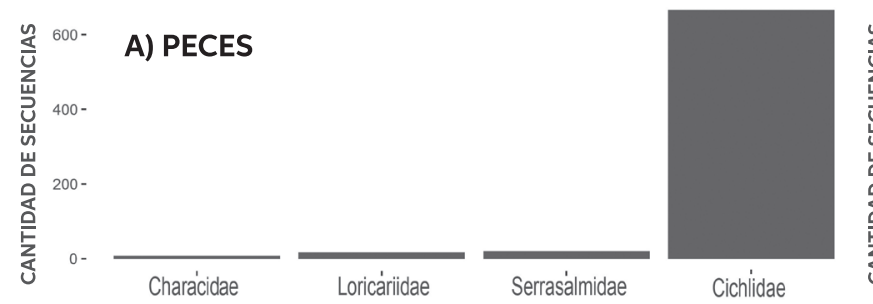

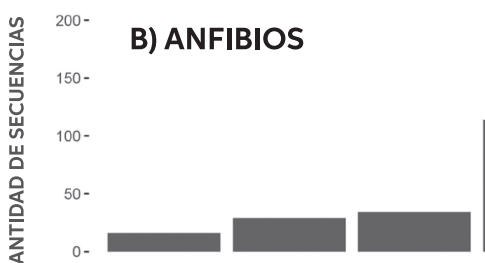

Leptodactylidae Aromobatidae Microhylidae

C) REPTILES
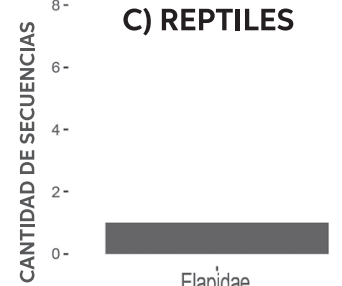

Elapidae

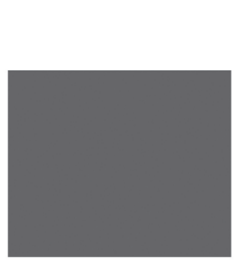

Iguánidae
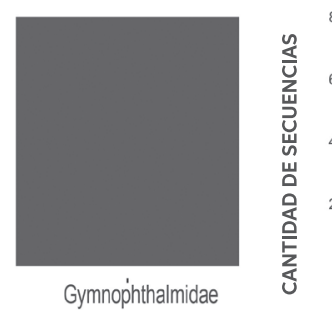

80- D) AVES

$40-$

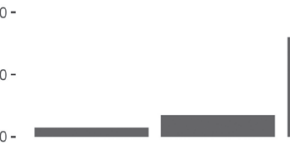

Pipridae

Trogonidae
Cotinguidae

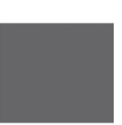

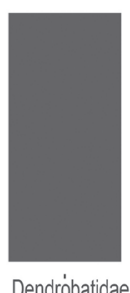

Hylidae

Bufónidae Dendróbatidae

E) MAMIFEROS

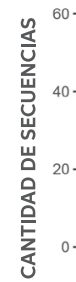

Cyclopjedidae
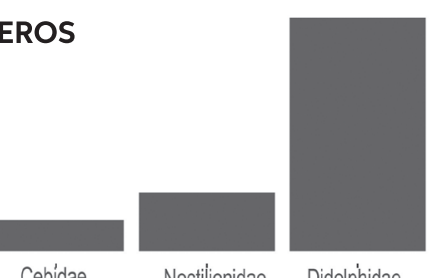

Didelphidae

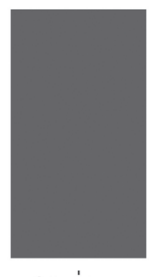

Cricetidae

Figura 3: Abundancia de secuencias genéticas por Familias de vertebrados en la región Loreto, Perú. 


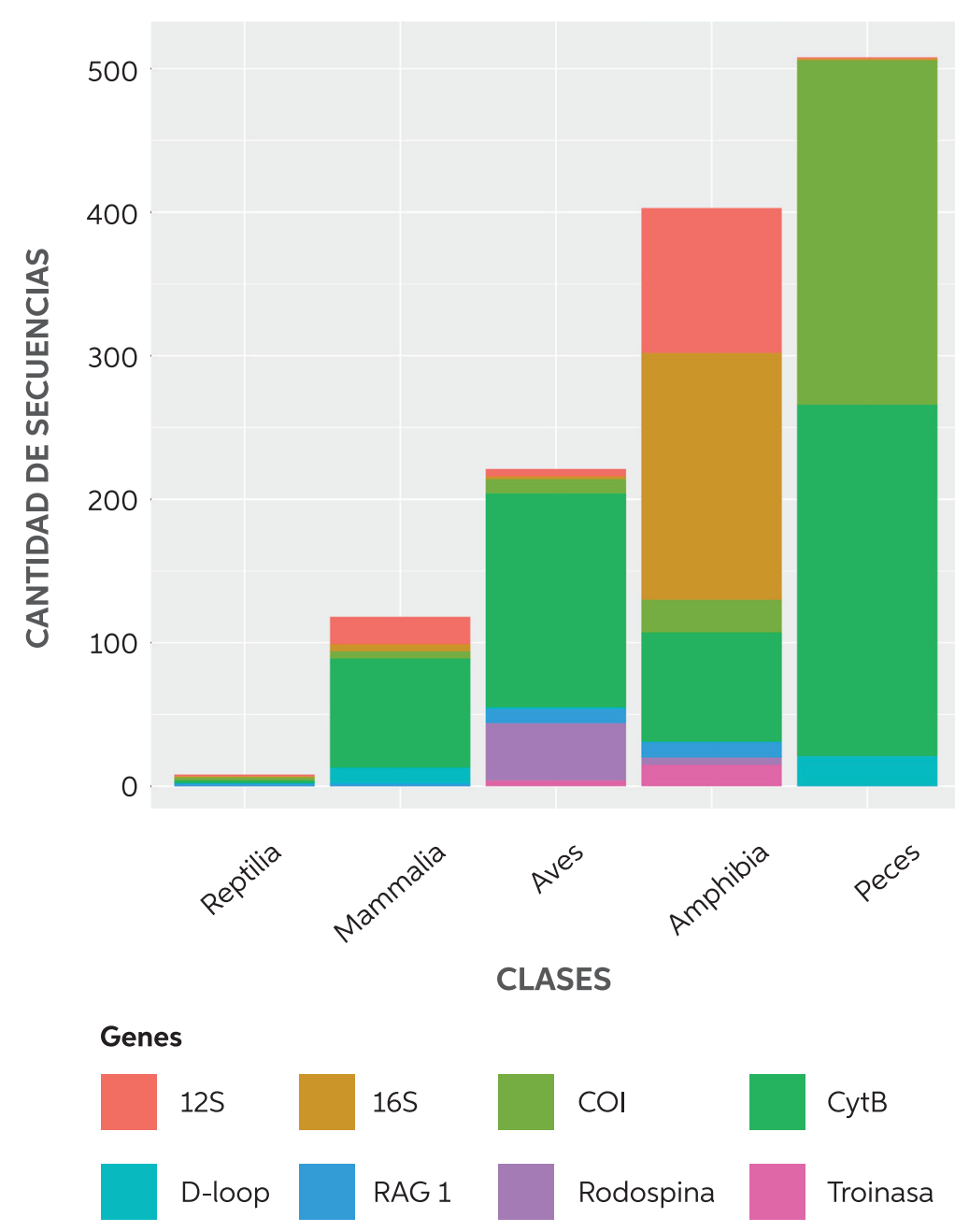

Figura 4: Abundancia de marcadores moleculares secuenciados de vertebrados de la región Loreto, Perú.

A nivel de especies, para Peces, las especies que presentaron un mayornúmerode secuenciasfuero Apistograma spp (666). Para la Clase Amphibia la especie con mayor cantidad de secuencias fue Amazophrynella amazonicola (80). La Clase Reptilia presento mayor representatividad por la especie Cercosaura oshaughnessyi (5). Para Aves, la especie más secuenciada fue Capito wallacei (24). La Clase Mammalia presento secuencias de la especie Neacomys sp. (21) como más abundante.

La cantidad y tipo de marcadores moleculares variaron por cada grupo de vertebrados. En caso de Peces el marcador mitocondrial Cytb (245) fue el más frecuente, seguido COI (241). Dentro de la Clase Amphibia el gen 16S fue el más secuenciado (207), seguido por el gen 12S (148). En Reptilia el marcador CytB fue el más abundante (3) seguido por COI (2). En Aves fue el gen mitocondrial CytB (149) fue el más estudiado, seguido por el gen nuclear rodopsina (40). En Mammalia el gen mitocondrial CytB (86) fue el más reportado, seguido por $12 \mathrm{~S}$ (19) (Figura 4).

Respecto a los especímenes de la fauna silvestre de interés comercial y para fines de conservación no existen reportes de secuencias genéticas. Por ejemplo, no se registraron secuencias en Peces (Arapaima gigas "Paiche), en reptiles (Podocnemis expansa "Charapa", $P$. unifilis "Taricaya", Caiman cocodrilo "caimán blanco" y Caiman niger "caimán negro") ni en mamíferos (Pecari tajacu "Huangana", Tayassu pecari "puerco del campo", Tapirus terrestres "Sachavaca", Mazama americana "venado").

Comparando el número de secuencias genéticas reportadas por regiones "Amazónicas" del Perú (Figura 5), Loreto posee un mayor número de secuencias (1 960), seguido por San Martin (249), Madre de Dios (153) y Ucayali (69).

\section{DISCUSIÓN}

Los resultados muestran que el número de secuencias genéticas reportadas para la región Loreto son escasas en comparación de la vasta diversidad reportada. Existe un gran vacío de información genética de diversos taxas de vertebrados en diferentes niveles taxonómicos, por lo que urge realizar investigaciones a fin de cubrir estos vacíos del conocimiento a nivel 
de procesos genético-evolutivos, detección de especies crípticas, estado genético de poblaciones, identificación de especies nuevas e inferencia de procesos filo y biogeográficos en todos los grupos taxonómicos.

A nivel taxonómica de Clase, nuestros datos muestran obvias diferencias en la abundancia de secuencias genéticas (ver Figura 1). Estos resultados indican la existencia de una mayor cantidad de estudios genéticos relacionados a especies de la Clase Peces y anfibios, y en menor proporción aves y mamíferos y en menor escala reptiles. Asimismo, se evidencia una acumulación diferencial de secuencias genéticas a nivel de Orden (Figura 2), como por ejemplo Cichliformes (Peces), Anura (Amphibia), Squamata (Reptilia), Passeriformes (Aves) y Rodentia (Mammalia) y en otros casos, indican la ausencia total de secuencias genéticas para algunos grupos como Apoda y Caudata (Amphibia) y Chelonia y Crocodrilia (Reptilia) y de igual forma para el nivel taxonómico de Familias (Figura 3).

A pesar de la alta diversidad de especies en Loreto, el número de especies que poseen secuencias genéticas registradas se encuentra ampliamente desproporcional con relación al número de especies registradas. Como por ejemplo 93 especies de la Clase Peces poseen secuencias nucleotídicas vs. 800 especies taxonómicamente registradas (Ortega et al., 2012); 64 vs. 216 de anfibios (Pitman et al., 2013); 7 vs. 170 de reptiles (Pitman et al., 2013); 105 vs. 914 de aves y 34 vs. 267 de mamíferos (Pitman et al., 2013). La ausencia de estos datos genéticos en la mayoría de las especies de vertebrados en Loreto no permite una estimación apropiada de la diversidad biológica como reportado en otros estudios (Fouquet et al., 2007; Angulo \& Icochea, 2010; Fouquet et al., 2014; Rowley et al., 2015; Ferrão et al., 2016).

Con relación a los genes más secuenciados para estudios genéticos en Loreto, es posible

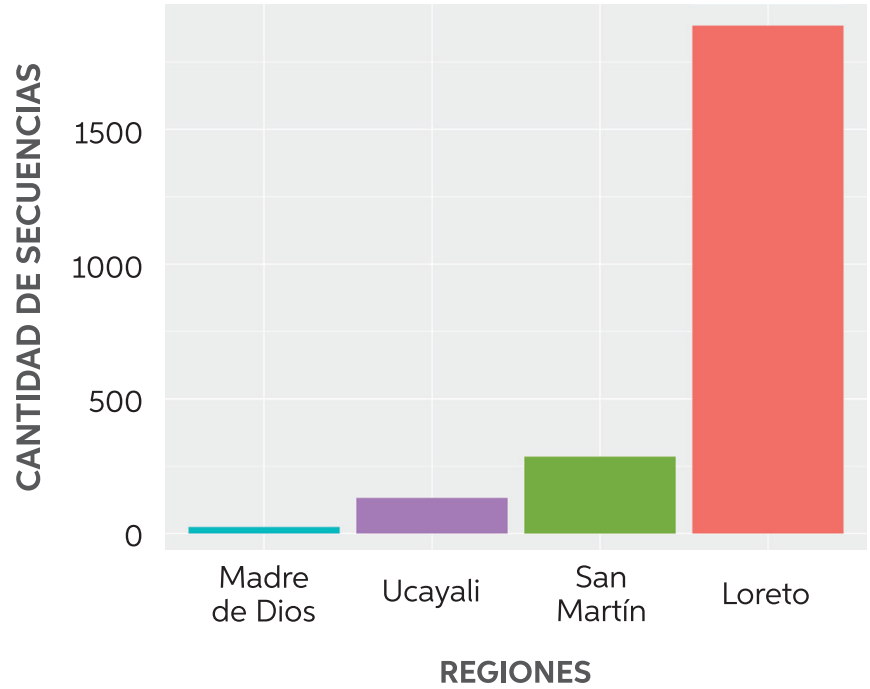

Figura 5: Abundancia de secuencias genéticas de vertebrados por regiones Amazónicas del Perú.

identificar que cada grupo de vertebrado presentó variaciones asociados al tipo de gen utilizado (Figura 4). Estos resultados están relacionados a la confiabilidad del marcador molecular usado como código de barras de DNA para los organismos en estudio (Hebert et al., 2003). Por ejemplo, para Amphibia el gen más usado en estudios genéticos a nivel global es el gen 16S (Vences et al., 2005), CytB en aves (Aleixo, 2007) y mamíferos (Bradley, 2001) y los genes COI y CytB en Peces (Ward et al. 2009).

Por otra parte la búsqueda de secuencias genéticas en el GenBank de especies de interés comercial y para la conservación de Loreto, como el "Paiche”, “Charapa”, “Caiman blanco", fue infructuosa. La ausencia de estos datos muestra la necesidad de complementar los diversos estudios ecológicos y de desarrollo sustentable con datos genéticos (secuencias mitocondriales, microsatélites, SNPs), a fin de realizar una evaluación integral de dinámica poblacional de estas especies, así como realizado en otros estudios con Arapaima gigas "Paiche" (Hrbek et al., 2005), Saguinus bicolor "Sauim de Coleira" (Farias et al., 2015), Trichechus inunguis "Manati" 
(Cantanhede et al., 2005), Caiman niger "Caiman negro" (de Thoisy et al., 2006).

\section{PERSPECTIVAS DE ESTUDIOS GENÉTICOS DE VERTEBRADOS EN LORETO, PERÚ}

A pesar de poseer una mayor cantidad de secuencias en territorios "Amazónicos" del Perú (Figura 5), nuestros resultados estiman que en comparación con las especies taxonómicamente registradas (ver Ortega et al., 2012 para peces y Pitman etal., 2013 para otros vertebrados) apenas $19,44 \%$ de especies de vertebrados en Loreto poseen datos genéticos. Por tanto, enriqueciendo esa base de datos se podrán realizar varias aplicaciones prácticas, desde la conservación de la diversidad biológica hasta su uso para estudios de mejoramiento genético, (Crandall et al., 2000; Benirschke, 2006).

Es conocido que la diversidad biológica se encuentra generalmente subestimada en los trópicos (Fouquet et al., 2007) siendo necesario estudios exploratorios de colecta de material biológico y estandarización de protocolos de purificación de DNA. La producción de estos datos genéticos permitirán establecer líneas base de diversidad e inferir procesos evolutivos (Benirschke, 2006; Carstens et al., 2013; De Queiroz, 2007; Leite \& Rogers, 2013).

Las secuencias genéticas de los vertebrados en Loreto pueden ser utilizados para aplicaciones forenses usando los códigos de barras de DNA (Alcantara \& Yambot 2016; Mishra et al., 2017). Esta metodología es conocida para la identificación de especies amenazas o en peligro, que fueron cazados ilegalmente y transportados por el comercio ilegal (Galimberti et al., 2015). Pero la ausencia de datos genéticos de especies de interés comercial dificultaría deducir su procedencia geográfica, de esta manera la creación de un banco de datos genéticos enriquecido para estas especies es indispensable para la lucha contra el tráfico ilegal de animales silvestres.

En términos de conservación de la diversidad biológica, las metodologías moleculares pueden permitir la identificación de "grupos" genéticos que con tendencia a ser conservados de forma diferentes en relación a otras poblaciones (Ryder, 1986). Estas metodologías permiten la creación de Unidades Evolutivamente SignificantesEvolutionary Significant Unit-ESU (Ryder, 1986) y Unidades de Conservación- Unit of conservation (Dodson et al., 1998). Poblaciones de especies genéticamente diferentes de otras que todavía no llegaron al status de especies, pueden presentar diferente ecología, morfología o comportamiento, siendo necesario ser conservados independientemente de otras poblaciones. En ese sentido, muchas de las iniciativas de conservación en Loreto pueden verse comprometidas si no son estudiadas al mismo tiempo las fuerzas y procesos evolutivos que modifican la dinámica espacial y temporal de las poblaciones naturales en ambientes diferentes.

Para fines de mejoramiento genético los análisis de estructuración poblacional son fundamentales (Wuyun et al., 2015). Los clados obtenidos con estos análisis permiten agrupar diferentes poblaciones de la misma especie en grupos estructurados biológicamente. Una vez que los grupos biológicos son identificados, es necesario estudiar aspectos ecológicos de estas poblaciones (medidas, pesos, rangos de resistencia y adaptabilidad a diferentes presiones ambientales, tipo de agua, profundidad, luminosidad, resistencia a $\mathrm{pH}$, etc.) para seleccionar aquellas que presenten características ventajosas. Asimismo, se debe garantizar el cruce entre estos grupos, seleccionando individuos que poseen caracteres de intereses para la crianza y al mismo tiempo reduciendo la endogamia. 
Adicionalmente, con el desarrollo de plataformas tecnologías de secuenciamiento de próxima generación, el número de secuencias de vertebrados disponibles, así como de otros organismos aumentará exponencialmente en un breve periodo de tiempo. Estas nuevas plataformas como Roche 454, SOLID, Illumina, Ion torrent, PacBio SMRT, HeliScope, pueden generar de miles a millones de secuencias (Mardis, 2013; Mutz et al., 2013; Shokralla et al., 2012). Como ejemplo para Loreto es el caso de Lepidothrix coronata (Aves: Pipridae), así mismo, se ha adaptado la tecnología de NGS para estudiar la dinámica reproductiva de Siluriformes, a través de la identificación molecular masiva de sus larvas mediante Metabarcoding (Maggia et al., 2016), estos dos ejemplos forman parte de otras especies Amazónicas que poseen datos genómicos (p.ej., De Carvalho et al., 2017; Martínez et al., 2017).

Estas tecnologías, juntamente con otras herramientas Estas nuevas plataformas tecnológicas de secuenciamiento masivo, junto con análisis bioinformáticos y el empleo de herramientas moleculares nos permitirá la colección y manipulación genética de los recursos naturales basados en principios biotecnológicos y al mismo tiempo contribuirá con el mejoramiento genético de varios organismos de interés comercial para la acuicultura, zoocrianza in situ y ex situ y otras alternativas de desarrollo social, económico y ambiental.

\section{BIBLIOGRAFIA CITADA}

Alcantara, S.G.; Yambot, A. V. 2016. DNA barcoding of commercially important Grouper species (Perciformes, Serranidae) in the Philippines. Mitochondrial DNA Part A DNA Mapping, Sequencing and Analysis, 27(6): 3837-3845. https://doi.org/10.3109/19401736.2014.95 8672

Angulo, A.; Icochea, J. 2010. Cryptic species complexes, widespread species and conservation: lessons from Amazonian frogs of the Leptodactylus marmoratus group (Anura: Leptodactylidae). Systematics and Biodiversity, 8: 357-370. https://doi.org/10.1080/147720 00.2010 .507264

Avise, J.C.; Arnold, J.; Ball, R.M.; Lambt, T.; Neigel, J.E.; Carol, A.; Saunders, N.C. 1987. Intraspecific phylogeography: The Mitochondrial DNA Bridge Between Population Genetics and Systematics. Annual Review of Ecology and Systematics, 18: 489-522.

Benirschke, K., 2006. Conservation and Genetics of Populations. Journal of Heredity, 97(6): 612-613. https://doi.org/10.1093/jhered/ esl039

Benson, D.A.; Cavanaugh, M.; Clark, K.; KarschMizrachi, I.; Lipman, D.J.; Ostell, J.; Sayers, E.W. 2013. GenBank. Nucleic Acids Research, 41: 3642. https://doi.org/10.1093/nar/gks1195

Bickford, D.; Lohman, D.J.; Sodhi, N.S.; Ng, P.K.L.; Meier, R.; Winker, K.; Ingram, K.K.; Das, I. 2007. Cryptic species as a window on diversity and conservation. Trends in Ecology and Evolution, 22: 148-55. https://doi.org/10.1016/j. tree.2006.11.004

Cantanhede, A.M.; Da Silva, V.M.F.; Farias, I.P.; Hrbek, T.; Lazzarini, S.M.; Alves-Gomes, J. 2005. Phylogeography and population genetics of the endangered Amazonian manatee, Trichechus inunguis Natterer, 1883 (Mammalia, Sirenia). Molecular Ecology, 14(2): 401-13. https://doi. org/10.1111/j.1365-294X.2004.02413.x

Carstens, B.C.; Pelletier, T.A.; Reid, N.M.; Satler, J.D. 2013. How to fail at species delimitation. Molecular Ecology. 22: 4369-4383. https:// doi.org/10.1111/mec.12413 
Cozzuol, M.A.; Clozato, C.L., Holanda, E.C.; Rodrigues, F.H.G.; Nienow, S.; de Thoisy, B.; Redondo, R.A.F.; Santos, F.R. 2013. A new species of tapir from the Amazon. Journal of Mammalogy, 94(6): 1331-1345. https://doi. org/10.1644/12-MAMM-A-169.1

Crandall, K.A.; Bininda-Emonds, O.R.R.; Mace, G.M.; Wayne, R.K. 2000. Considering evolutionary processes in conservation biology. Trends in Ecology and Evolution, 15(7): 290-295. https://doi.org/10.1016/ S0169-5347(00)01876-0

de Carvalho, V.T.; Martínez, J.G.; HernándezRangel, S.M.; Astolfi-Filho, S.; Vogt, R.C.; Farias, I.P.; Hrbek, T. 2017. Giving IDs to turtles: SNP markers for assignment of individuals to lineages of the geographically structured Phrynops geoffroanus (Chelidae: Testudines). Conservation Genetics Resources, 9: 157-163. https://doi.org/10.1007/s12686-016-0626-8 De Queiroz, K. 2007. Species concepts and species delimitation. Systematic. Biology, 56: 879-86. https://doi. org/10.1080/10635150701701083

de Thoisy, B.; Hrbek, T.; Farias, I.P.; Vasconcelos, W.R.; Lavergne, A. 2006. Genetic structure, population dynamics, and conservation of Black caiman (Melanosuchus niger). Biological Conservation, 133: 474-482. https://doi. org/10.1016/j.biocon.2006.07.009

Dodson, J.J.; Gibson, R.J.; Cunjak, R.; Friedland, K.D.; de Leaniz, C.G.; Gross, M.R.; Newbury, R.; Nielsen, J.L.; Power, M.E.; Roy, S. 1998. Elements in the development of conservation plans for Atlantic salmon (Salmo salar). Canadian Journal of Fisheries and Aquatic Sciences, 55: 312-323. https://doi.org/10.1139/cjfas55-S1-312

Farias, I.P.; Santos, W.G.; Gordo, M.; Hrbek, T. 2015. Effects of Forest Fragmentation on Genetic Diversity of the Critically Endangered Primate, the Pied Tamarin (Saguinus bicolor):
Implications for Conservation. Journal of Heredity, 106: 512-521. https://doi. org/10.1093/jhered/esv048

Fearnside, P.M. 2006. Dams in the Amazon: Belo Monte and Brazil's hydroelectric development of the Xingu River Basin. Environmental Management, 38(1): 16-21. https://doi. org/10.1007/s00267-005-0113-6

Fearnside, P.M., 2005. Deforestation in Brazilian Amazonia: History, rates, and consequences. Conservation Biology, 19(3): 680-688. https:// doi.org/10.1111/j.1523-1739.2005.00697.x

Fearnside, P.M. 1996. Amazonian deforestation and global warming: carbon stocks in vegetation replacing Brazil's Amazon forest. Forest Ecology and Management, 80: 1-3. https://doi.org/10.1016/03781127(95)03647-4

Ferrão, M.; Colatreli, O.; De Fraga, R.; Kaefer, I.L.; Moravec, J.; Lima, A.P. 2016. High species richness of scinax treefrogs (hylidae) in a threatened amazonian landscape revealed by an integrative approach. PLoS One 11, 1-16. https://doi.org/10.1371/journal. pone.0165679

Florio, A.M.; Ingram, C.M.; Rakotondravony, H.A.; Louis, E.E.; Raxworthy, C.J. 2012. Detecting cryptic speciation in the widespread and morphologically conservative carpet chameleon (Furcifer lateralis) of Madagascar. Journal of Evolutionary Biology, 25: 13991414. https://doi.org/10.1111/j.14209101.2012.02528.x

Fouquet, A.; Gilles, A.; Vences, M.; Marty, C.; Blanc, M.; Gemmell, N.J. 2007. Underestimation of species richness in neotropical frogs revealed by mtDNA analyses. PLoS One 2, e1109. https:// doi.org/10.1371/journal.pone.0001109

Fouquet, A.; Santana, C.; Baptista Haddad, C.; Pech, N.; Trefaut, M. 2014. Species delimitation, patterns of diversification and historical biogeography of the Neotropical frog genus 
Adenomera (Anura, Leptodactylidae). Journal of Biogeography, 41: 855-870. https://doi. org/10.1111/jbi.12250

García-Dávila, C.; Castro-Ruiz, D.; Sanches, H.; Ismiño, R.; Rengifo, D.; García, A.; Tello, S.; Chota-Macuyama, W.; Duponchelle, F.; Renno, J.-F. 2014. Ictioplanct diversity in the Curaray, Arabela and Napo river (Peruvian Amazon). Folia Amazonica, 23 (1):67-78.

García-Dávila, C.; Castro-Ruiz, D.; Renno, J.F.; Chota-Macuyama, W.; Carvajal-Vallejos, F.; Sanchez, H.; Angulo, C.; Nolorbe, C.; Alvarado, J.; Estivals, G.; Núñez-Rodríguez, J.; Duponchelle. F. 2015. Using barcoding of larvae for investigating the breeding seasons of pimelodid catfishes from the Marañon, Napo and Ucayali rivers in the Peruvian Amazon. Journal of Applied Ichthyology, 31: 40-51.

Galimberti, A.; Sandionigi, A.; Bruno, A.; Bellati, A.; Casiraghi, M. 2015. DNA barcoding in mammals: What's new and where next? Italian Journal of Mammalogy, 26(5): 13-24. https:// doi.org/10.4404/hystrix-26.1-11347

Gehara, M.; Crawford, A.J.; Orrico, V.G.D.; Rodríguez, A.; Lötters, S.; Fouquet, A.; Barrientos, L.S.; Brusquetti, F.; De La Riva, I.; Ernst, R.; Urrutia, G.G.; Glaw, F.; Guayasamin, J.M.; Hölting, M.; Jansen, M.; Kok, P.J.R.; Kwet, A.; Lingnau, R.; Lyra, M.; Moravec, J.; Pombal, J.P.; Rojas-Runjaic, F.J.M.; Schulze, A.; Señaris, J.C.; Solé, M.; Rodrigues, M.T.; Twomey, E.; Haddad, C.F.B.; Vences, M.; Köhler, J. 2014. High levels of diversity uncovered in a widespread nominal taxon: Continental phylogeography of the neotropical tree frog Dendropsophus minutus. PLoS One 9. https://doi.org/10.1371/journal. pone.0103958

Ortega, J.; Trevejo, E.; Correa, A.; Cortijo, V.; Meza, J.E. 2012. Lista anotada de los peces neotropicales de aguas continentales del Perú, Memorias Museo de Historia Natural, U.N.M.S.M. Lima. 81pp.
Hebert, P.D.N.; Cywinska, A.; Ball, S.L.; deWaard, J.R. 2003. Biological identifications through DNA barcodes. Proceedings of the Royal Society B: Biological Science, 270: 313-21. https://doi. org/10.1098/rspb.2002.2218

Hedrick, P.W.; Miller, P.S. 1992. Conservation genetics: techniques and fundamentals. Ecological Applications, 2(1): 30-46. https:// doi.org/10.2307/1941887

Hrbek, T.; da Silva, V.M.F.; Dutra, N.; Gravena, W.; Martin, A.R.; Farias, I.P. 2014. A New Species of River Dolphin from Brazil or: How Little Do We Know Our Biodiversity. PLoS One 9, e83623. https://doi.org/10.1371/journal. pone.0083623

Hrbek, T.; Farias, I.P.; Crossa, M.; Sampaio, I.; Porto, J.I.R.; Meyer, A. 2005. Population genetic analysis of Arapaima gigas, one of the largest freshwater fishes of the Amazon basin: Implications for its conservation. Animal Conservation, 8: 297-308. https://doi. org/10.1017/S1367943005002210

Jenkins, C.N.; Pimm, S.L.; Joppa, L.N. 2013. Global patterns of terrestrial vertebrate diversity and conservation. PNAS, 110: 2602-2610. https:// doi.org/10.1073/pnas.1302251110

Leite,R.N.; Rogers, D.S.2013.RevisitingAmazonian phylogeography: Insights into diversification hypotheses and novel perspectives. Organisms Diversity and Evolution, 13(4): 639-664. https://doi.org/10.1007/s13127-013-0140-8 Maggia, M. E.; Vigouroux, Y.; Renno, J. F.; Duponchelle, F.; Desmarais, E.; Nunez, J.; Garcia-Davila, C.; Carvajal-Vallejos, F. M.; Paradis, E.; Martin, J. F.; Mariac, C. 2017. DNA Metabarcoding of Amazonian Ichthyoplankton Swarms. PLOS ONE. https://doi.org/10.1371 / journal.pone.0170009

Mardis, E.R. 2013. Next-Generation Sequencing Platforms.AnnualReviewofAnalyticalChemistry, 6: 287-303. https://doi.org/10.1146/ annurev-anchem-062012-092628 
Martínez, J.G.; Machado, V.N.; Caballero-Gaitán, S.J.; Santos, M.; Alencar, R.M.; Escobar M.D.; Hrbek, T.; Farias, I.P. 2017. SNPs markers for the heavily overfished tambaqui Colossoma macropomum, a Neotropical fish, using next-generation sequencing-based de novo genotyping. Conservation Genetics Resources, 9: 23-33. https://doi.org/10.1007/s12686016-0610-3

Mishra, P.; Kumar, A.; Sivaraman, G.; Shukla, A.K.; Kaliamoorthy, R.; Slater, A.; Velusamy, S. 2017. Character-based DNA barcoding for authentication and conservation of IUCN Red listed threatened species of genus Decalepis (Apocynaceae). Scientific Reports, 7: 14910. https://doi.org/10.1038/s41598-01714887-8

Mittermeier, R. A.; Myers, N.; Thomsen, J. B.; da Fonseca, G. A. B.; Olivieri, S. 1998. Biodiversity hotspots and major tropical wilderness areas: approaches to setting conservation priorities. Conservation Biology, 12, 516-520.

Mutz, K.O.; Heilkenbrinker, A.; Lönne, M.; Walter; J.G.; Stahl, F. 2013. Transcriptomeanalysis using next-generation sequencing. Current Opinion in Biotechnology, 24(1): 22-30. https://doi. org/10.1016/j.copbio.2012.09.004

Pitman, N., Gagliardi Urrutia, G., Jenkins, C., 2013. La Biodiversidad de Loreto, Perú. El conocimiento actual de la diversidad de plantas y vertebrados terrestres. Center for International Enviromental Law, USA. 21pp. https://doi.org/10.13140/2.1.2641.4406

Rojas, R.R.; Chaparro, J.C.; De Carvalho, V.T.; Ávila, R.W.; Farias, I.P.; Hrbek, T.; Gordo, M. 2016. Uncovering the diversity inside the Amazophrynella minuta complex: integrative taxonomy reveals a new species of Amazophrynella (Anura, Bufonidae) from southern Peru. Zookeys, 71: 43-71. https:// doi.org/10.3897/zookeys.563.6084

Rowley, J.J.L., Tran, D.T.A., Frankham, G.J., Dekker, A.H., Le, D.T.T., Nguyen, T.Q., Dau, V.Q., Hoang, H.D., 2015. Undiagnosed cryptic diversity in small, microendemic frogs (Leptolalax) from the Central Highlands of Vietnam. PLoS One 10. https://doi.org/10.1371/journal. pone.0128382

Ryder, O.A. 1986. Species conservation and systematics: the dilemma of subspecies. Trends in Ecology and Evolution, 1(1): 9-10. https:// doi.org/10.1016/0169-5347(86)90059-5

Shanee, N. 2012. Trends in local wildlife hunting, trade and control in the Tropical Andes Biodiversity Hotspot, northeastern Peru. Endangered Species Research, 19(2):177-186. https://doi.org/10.3354/esr00469

Shokralla, S.; Spall, J.L.; Gibson, J.F.; Hajibabaei, M. 2012. Next-generation sequencing technologies for environmental DNA research. Molecular Ecology, 21(8): 1794-1805. https:// doi.org/10.1111/j.1365-294X.2012.05538.X

Vences, M.; Thomas, M.; Van der Meijden, A.; Chiari, Y.; Vieites, D.R. 2005. Comparative performance of the $16 \mathrm{~S}$ rRNA gene in DNA barcoding of amphibians. Frontiers of Zoology, 2(1): 5. https://doi.org/10.1186/1742-9994$2-5$

Wuyun, T.; Amo, H.; Xu, J.; Ma, T.; Uematsu, C.; Katayama, H. 2015. Population structure of and conservation strategies for wild Pyrus ussuriensis Maxim. in China. PLoS One, 10(8): e0133686. https://doi.org/10.1371/journal. pone.0133686

Recibido: 1 de octubre de 2018 Aceptado para publicación: 1 de diciembre de 2018 